\title{
INVESTIGACIÓN Y DESARROLLO DE TRES PRODUCTOS DEL JABONCILLO (SAPINDUS SAPONARIA L.) COMO BASE PARA SU INDUSTRIALIZACIÓN
}

\author{
Study and development of three jaboncillo (Sapindus saponaria L.) \\ products as grounding in its industrialisation
}

Palabras clave: Sapindus saponaria, sapogeninas, hederagenina, goma, aceite.

Keywords: Sapindus saponaria, sapogenins, hederagenin, gum, oil.
Henry A. Flechas C. $^{1}$

Camilo Aragón $D^{2}$

Nelly B. Morales ${ }^{2}$

P. John A. Jiménez G. ${ }^{2}$

\section{RESUMEN}

Esta investigación comprendió el estudio químico, fitoquímico y el escalado industrial, en planta piloto, de tres productos de la especie Sapindus saponaria conocida comúnmente en Colombia como jaboncillo, Michu. Este trabajo hace parte de un proyecto interdisciplinar encaminado a aprovechar y comercializar sus productos derivados: aceite, goma y sapogeninas triterpenoidales. La obtención de estos tres productos se llevó a cabo mediante procesos físicos y químicos tanto en laboratorio (Fase 1), como en la etapa de escalado industrial (Fase 2). En la etapa de laboratorio se estudiaron y evaluaron diferentes métodos de extracción y purificación, al igual que los correspondientes métodos de análisis químico para tres procedencias (Palmira, Valle; Viotá, Cundinamarca, y Santa María, Huila). Con base en los estudios de laboratorio se diseñaron los procesos de extracción y purificación en planta piloto suministrada por la empresa QUINACOL LTDA., cuya razón social es la producción, investigación y desarrollo de productos para la industria fitofarmacéutica. Los productos desarrollados fueron caracterizados por métodos de análisis instrumental de resonancia magnética nuclear (RMN de ${ }^{1} \mathrm{H}$ y ${ }^{13} \mathrm{C}$ ), cromatografía de gases con detector de ionización de llama (CG), espectroscopia infrarroja (IR) y técnic as de análisis químico específicas para estos productos.
El fruto maduro de Santa María presentó un mayor rendimiento de aceite y goma; así como el fruto verde de Viotá, en una sustancia denominada Ss1, posiblemente hederagenina. De los productos obtenidos en la etapa piloto, el aceite presentó un alto porcentaje en ácidos grasos insaturados y la goma mostró una alta capacidad espesante en soluciones acuosas. Del fruto de esta especie se podrían comercializar el aceite y la goma, pues su composición y características, así como su rendimiento, cumplen con los principales parámetros de mercadeo, para lo cual es necesario realizar estudios de prefactibilidad industrial.

\begin{abstract}
This research included the chemical and phytochemical studies and the industrial scaling in pilot plant of three products of Sapindus saponaria, commonly known as jaboncillo, Michu. This work is part of an interdisciplinary research project aimed at the exploitation and commercialization of its harvested products: oil, gum, and triterpenoidal sapogenins. These three products were obtained through physical and chemical processes both in laboratory (phase 1) and in the industrial scaling stage (phase 2). During the laboratory stage different methods of extraction and purification were studied and assessed, along with the corresponding methods of chemical analysis for the three origins
\end{abstract}

1 Proyecto Curricular de Ingeniería Forestal, Universidad Distrital Francisco José de Caldas. Correspondencia: hflechascorredor@gmail.com

2 Proyecto Curricular de Licenciatura en Química, Universidad Distrital Francisco José de Caldas. camicardu@gmail.com 
(Palmira, Valle; Viotá, Cundinamarca, and Santa María, Huila). Based on laboratory studies, extraction and purification processes were designed in a pilot plant supplied by the QUINACOL LTDA. company, whose business is the production, research and development of products for the phytopharmaceutical industry. Developed products were characterized by methods of instrumental analysis of nuclear magnetic resonance (1 H NMR and 13c), flame ionization detector gas chromatography (GC), infrared spectroscopy (IR) and specific chemical analysis techniques designed for these products. The mature fruit of Santa María presented increased performance of oil and rubber; as well as the green fruit of Viotá, in a substance called Ss1, possibly hederagenina. Of the products obtained in the pilot phase, oil presented a high percentage of unsaturated fatty acids and rubber showed a high thickening capacity in aqueous solutions. Oil and rubber could be marketed from this species' fruit, because its composition and characteristics, as well as its performance, meets the main parameters of marketing, for which studies on industrial perfectibility are needed.

\section{INTRODUCCIÓN}

El presente artículo describe el estudio químico, fitoquímico y de planta piloto de la especie Sapindus saponaria L. como parte del proyecto multidisciplinar: "Investigación y desarrollo de un proyecto empresarial encaminado a obtener y comercializar productos derivados de la especie Sapindus saponaria". Esta especie también se conoce como jaboncillo o chumbimbo y pertenece a la familia Sapindaceae, es un árbol mediano a grande que puede alcanzar hasta $20 \mathrm{~m}$ de altura. Las flores son pequeñas, de pétalos blancos y muy fragantes. Los frutos son drupas esféricas, con pericarpio amarillo y translúcido; las semillas son negras y redondas. Es apropiado como barrera, especialmente contra vientos fuertes; como sombrío, en separadores amplios, en retiros de quebradas y en programas de reforestación. La madera es muy dura y pesada, de textura áspera y poco durable a la intemperie, es un árbol de la América tropical.

El fruto de la $S$. saponaria ha sido estudiado por varios investigadores (Riveiro 1995, Sandoval 2003,
Hess 2003). En Colombia, Abreu \& Carulla (2003) encontraron aplicaciones de éste como suplemento alimenticio en la dieta de bovinos. Sin embargo, no existen publicaciones sobre los productos, ni aprovechamiento. Por lo tanto, este estudio exploratorio buscó aprovechar el fruto en sus diferentes estados de maduración, mediante la obtención de aceite, goma y hederagenina (productos de alta demanda industrial), comparando distintos métodos de extracción y purificación en laboratorio que pudieran ser aplicados a nivel industrial.

Esta investigación fue financiada por el centro de investigaciones y desarrollo científico (CIDC) de la Universidad Distrital Francisco José de Caldas, que unió el trabajo de los grupos de investigación: de Ingeniería Forestal, Productos y Procesos del Bosque (Proprobos); de Ingeniería Industrial, Gestión Empresarial e Innovación Tecnológica (GEIT), y Licenciatura en Química.

\section{METODOLOGÍA}

Para facilitar la obtención de los subproductos del fruto, la metodología se dividió en dos fases: la Fase 1 incluyó la caracterización morfológica de los frutos y su viabilidad germinativa al igual que la implementación de las técnicas de laboratorio para la extracción, purificación y análisis de los subproductos. De cada procedencia se tomaron tres muestras para obtener los tres subproductos de interés. Para la Fase 2, se escogieron los más adecuados de estos procesos para aplicarlos en las operaciones unitarias en escala piloto, tales como: molienda, extracción, filtración, hidrólisis, y evaporación, entre otras.

\section{FASE 1}

Los frutos se recolectaron en tres regiones del país donde se encontró una alta densidad poblacional de árboles. Estas regiones fueron Palmira, Valle; Santa María, Huila, y Viotá, Cundinamarca; los frutos se recolectaron entre los meses de julio y agosto de 2005 (el fruto verde el día 70-80 después de floración y el fruto maduro el día 88, Sánchez 2008). Los frutos se clasificaron y separaron de acuerdo al estado de maduración, se determinaron las caracte- 
rísticas morfológicas y el porcentaje de humedad (\% de humedad) por el método ASTM D 143903.

Los frutos sanos se despulparon manualmente, las semillas se lavaron y molieron en un molino universal manual. Para extraer el aceite, la torta se pesó en una balanza semianalítica, OHAUs Adventure TM. El aceite se extrajo con éter de petróleo en Soxhltes durante cinco horas. El extracto orgánico se filtró al vacío en un embudo Buchner con papel Whatmann No. 3, posteriormente se concentró en un evaporador rotatorio HEIDOLPH Laborota 4003, para luego ser deshidratado a $84^{\circ} \mathrm{C}$ durante $30 \mathrm{~min}$ en estufa de calentamiento (HEIDOLPH MR 3001). El aceite deshidratado se dejó enfriar y luego se pesó.

La goma se extrajo de la pulpa madura mediante dos procedimientos. En el primero (A) se pesaron $5 \mathrm{~g}$ de pulpa a la cual se agregaron $20 \mathrm{ml}$ de agua a $75^{\circ} \mathrm{C}$ por un periodo de $25 \mathrm{~min}$ con agitación periódica. Al final se procedió a separar la goma de la torta mediante filtración en caliente. Al extracto acuoso se le agregó isopropanol en una proporción 4:1 y se dejó en reposo durante 6 h hasta que las gomas se precipitaron. Luego se separaron del sobrenadante y se secaron en una estufa, posteriormente se pulverizaron y tamizaron. Durante el segundo procedimiento (B) se pesaron $5 \mathrm{~g}$ de pulpa madura a la que se le agregaron $10 \mathrm{ml}$ de ácido fosfórico al $10 \%$ a temperatura ambiente, y se dejó en reposo por $24 \mathrm{~h}$. Las gomas se separaron de la torta y se centrifugaron por $5 \mathrm{~min}$ a $6000 \mathrm{rpm}$. Luego se secaron en estufa, se molieron y tamizaron.

De la pulpa verde se extrajo la sapogenina triterpenoidal mediante tres procedimientos, dos de los cuales (Ps1 y Ps2) ya habían sido aplicados en la industria de las sapogeninas esteroidales (Sharapin 2000), como en el caso de la hecogenina y de la diosgenina a partir de los Agaves y las Dioscoreas, respectivamente. El tercer procedimiento (Ps3) se desarrolló en esta investigación para la extracción de sapogeninas triterpénicas.

En los diferentes métodos se utilizaron $200 \mathrm{~g}$ de pulpa seca y molida, siguiendo el siguiente procedimiento: el fruto se lavó y ablandó en agua por 3 días, una vez cumplido este tiempo se despulpó ma- nualmente. De la pulpa verde se tomaron 3 muestras (por procedencia), cada una de $200 \mathrm{~g}$, que se llevaron a digestión a $70^{\circ} \mathrm{C}$ con $\mathrm{NaCl}$ al $0.9 \%$ por $1 \mathrm{~h}$ (Marcano 1991). Terminada la digestión se prensó y filtró la pulpa en caliente. El extracto se fermentó por 4 días (completando 7 días totales el ablandamiento, la digestión y el fermentado). Luego de este tiempo, el sobrenadante se desechó y el precipitado (Torta 1) se llevó a hidrólisis por $4 \mathrm{~h}$ a reflujo en ácido sulfúrico diluido $(\mathrm{pH} 1$ mezcla torta-ácido). La mezcla se dejó en reposo por 12 h y el sobrenadante se desechó. El precipitado se llevó a una segunda hidrólisis por $4 \mathrm{~h}$ a reflujo en ácido sulfúrico, luego se dejó en reposo por 12 h y el sobrenadante se reutilizó en la primera hidrólisis de la segunda muestra. El precipitado (torta 3) se neutralizó con agua y bicarbonato de sodio al 10\%. Se secó en horno a $60^{\circ} \mathrm{C}$ y se extrajo a reflujo con metanol por $35 \mathrm{~min}$. El extracto metanólico se llevó a sequedad y se retomó con metanol analítico para ser pasado por medio filtrante de bentonita: carbón activado 1:1. La cristalización se llevó a cabo agregando la solución lentamente en agua.

\section{FASE 2}

Con base en los experimentos de laboratorio se identificaron las variables para diseñar los diferentes procesos $\mathrm{u}$ operaciones unitarias en la planta piloto. Se tomaron frutos de la mejor procedencia para extraer el aceite, las gomas y las sapogeninas. Se tomaron $12 \mathrm{~kg}$ de semillas despulpadas procedentes de Santa María a las cuales se les aplicó la prueba de viabilidad germinativa. Éstas se colocaron en agua, utilizando únicamente las que se sumergieron. Posteriormente se procedió a molerlas en un molino industrial de discos. Este material vegetal se pesó y se tamizó a través de mallas de $4 \mathrm{~mm}$ y $1 \mathrm{~mm}$ con el objeto de separar la harina de la testa. La semilla pulverizada se pesó nuevamente y se procedió a extraer con $5 \mathrm{~L}$ de Eter-pet a temperatura ambiente $\left(20 \pm 3^{\circ} \mathrm{C}\right)$ en una batería de percoladores de acero inoxidable por espacio de 3 h 20 min. El extracto etéreo se concentró por evaporación al vacío en un concentrador tipo Roberts a $40^{\circ} \mathrm{C}$ con una presión negativa de - 20 psi durante $55 \mathrm{~min}$. Al aceite obtenido se le eliminó el exceso de solvente en una estufa a $60^{\circ} \mathrm{C}$ por 30 minutos 
con agitación constante, luego se deshidrató a $84^{\circ} \mathrm{C}$ en horno de aire circulante por $90 \mathrm{~min}$. El aceite deshidratado se lavó centrifugándolo con agua (máximo $25 \%$ peso/volumen) a $70^{\circ} \mathrm{C}$ y neutralizando con solución de кон al $0.1 \%$ peso/volumen a $75^{\circ} \mathrm{C}$. Luego del lavado, se tomó un muestra de aceite (SMAC) que se secó en estufa al vacío y en campana de vidrio con petóxido de fósforo, luego se metiló y se analizó por cromatografía de gases en un cromatógrafo Hewlett Packard HP 6890, frente a patrones de ácidos grasos grado analítico, usando detector FID (Flame-Ionization Detector) y de conductividad térmica. El solvente utilizado en la fase móvil fue el heptano, usado frecuentemente en este tipo de análisis. El equipo se operó en un rango de temperatura entre 180 y $260^{\circ} \mathrm{C}$. Por otro lado, se determinó el índice de yodo bajo la Norma Técnica Colombiana NTC 283/1994 (Norma Técnica Colombiana, número de la norma/ año de la norma. Entiéndase igual para todas las NTC), la acidez con la NTC 218/1994, el índice de saponificación con la NTC 335/1994, la densidad con la NTC 336/2001 y el índice de refracción.

La extracción de goma se llevó a cabo utilizando los frutos maduros de la procedencia de Santa María, que se despulparon en un molino universal de $2 \mathrm{HP}$, resultando $6.8 \mathrm{~kg}$ de pulpa a la que se le realizaron dos extracciones sucesivas, la primera con 24.1 L de agua a $90^{\circ} \mathrm{C}$ por 45 min y la segunda con $23.11 \mathrm{~L}$ de agua a $100^{\circ} \mathrm{C}$ por $60 \mathrm{~min}$, ambas en un recipiente de acero inoxidable con agitación de 75 rpm. Los extractos se unieron en un solo volumen que se pasó por un filtro manga. Éste se concentró al $10 \%$ a $100^{\circ} \mathrm{C}$ por $16 \mathrm{~h} 20 \mathrm{~min}$ en un concentrador tipo Roberts de operación continua; luego, se llevó a sequedad en estufa de aire circulante a $94^{\circ} \mathrm{C}$ por $24 \mathrm{~h}$. Al extracto concentrado se le agregaron $18.5 \mathrm{~L}$ de isopropanol para extraer la goma (Abed 2002) luego se procedió a concentrar al vacío en un concentrador tipo Roberts, 20 psi a $80^{\circ} \mathrm{C}$ por $10 \mathrm{~h}$ $30 \mathrm{~min}$. Posteriormente se secó en horno de aire circulante a $105^{\circ} \mathrm{C}$ por $2 \mathrm{~h}$ y luego se pulverizó. $\mathrm{Al}$ extracto concentrado y a la goma pulverizada se les determinó: viscosidad, \% de humedad según la norma ASTM D 143903 y cenizas a $650^{\circ} \mathrm{C}$ por 12 horas. El porcentaje de taninos se determinó por colorimetría, según el siguiente procedimiento: una muestra de $50 \mathrm{ml}$ de goma al $2 \%$ en agua destilada se diluyó $1 / 200$. De esta dilución se tomó una alícuota de $25 \mathrm{ml}$ (concentración de goma $0.01 \%$ ) a la que se le agregó $1 \mathrm{ml}$ de reactivo fenol-folin y $10 \mathrm{ml}$ de reactivo carbonato tartrato, y se dejó en reposo por 30 min hasta su homogenización. La absorbancia máxima para taninos determinada fue de $730 \mathrm{~nm}$ en un equipo Perkin Elmer Lambda 10 (UV -VIS) con patrón de ácido tánico. Se realizó una curva de calibración en un Spectronic 20 con soluciones stock de ácido tánico, en concentraciones en un intervalo de $1 \times 10^{-4}$ a $5 \times 10^{-4}$ peso/volumen, usando como blanco $1 \mathrm{ml}$ de reactivo de fenol - folin y $10 \mathrm{ml}$ de carbonato tartrato en $25 \mathrm{ml}$ de agua destilada. La determinación cualitativa de azúcares se realizó por cromatografía en capa fina con sílica gel. La fase móvil consistió en una mezcla eleutrópica de acetato de etilo: isopropanol: agua (14:8:3), con patrones de fructosa, glucosa, galactosa, xilosa, ramnosa, manosa y ribosa, en solución al 1\%. Las placas se revelaron con vainillina en ácido sulfúrico y naftilamina en etanol al $2 \%$.

Para extraer las sapogeninas el fruto verde se separó en dos lotes, cada uno de ellos (PP1 - $11.5 \mathrm{~kg}$ y PP2-10.95 kg) se lavó y ablandó en un reactor con camisa de aceite térmico (PP1 a $103.8^{\circ} \mathrm{C}$ y 3 PSI por 31 min y pP 2 a $83^{\circ} \mathrm{C}$ y 1 PSI por $65 \mathrm{~min}$ ), y luego se despulpó por fricción en caliente y se pasó por dos tamices $(4 \mathrm{~mm}$ y $1 \mathrm{~mm})$. A la pulpa tamizada se le agregó agua destilada hasta obtener una pasta consistente que se dejó fermentar por espacio de 192 h. El precipitado se hidrolizó a reflujo con $\mathrm{H}_{2} \mathrm{SO}_{4}$ a $\mathrm{pH} 2$ en un reactor de acero inoxidable con tiempo de residencia de $230 \mathrm{~min}$. El hidrolizado se dejó en reposo para precipitar la fase sólida que se llevó a una segunda hidrólisis en $\mathrm{H}_{2} \mathrm{SO}_{4}\left(\mathrm{PP} 1\right.$ en $\mathrm{H}_{2} \mathrm{SO}_{4} 1 \mathrm{~N}$ por 215 min y $\mathrm{PP} 2 \mathrm{en}_{2} \mathrm{SO}_{4} 3 \mathrm{~N}$ por $85 \mathrm{~min}$ ). Los precipitados obtenidos se lavaron con agua y bicarbonato de sodio al $10 \%$, se secaron a temperatura ambiente por $13 \mathrm{~h}$ y luego en horno de aire circulante, PP 1 por $24 \mathrm{~h}$ a $36^{\circ} \mathrm{C}$ y PP 2 por $12 \mathrm{~h} 30 \mathrm{~min}$ a $36^{\circ} \mathrm{C}$. Luego se extrajeron en metanol a $40^{\circ} \mathrm{C}$. El extracto metanólico se concentró $1 / 10$ al vacío en el concentrador industrial. Las sapogeninas se cristalizaron con agua en un cristalizador piloto, se separaron de las aguas madre, se secaron y se disolvieron en acetona caliente. A esta solución se le 
adicionó cloroformo, formándose dos fases, luego se le adicionó agua, formándose una interfase con los cristales de sapogenina. Los cristales de sapogenina obtenidos de los lotes PP1 y PP2 se analizaron por cromatografía en capa fina en sílica gel con patrones de tigogenina y hecogenina, utilizando las siguientes mezclas eleutrópicas: diclorometano: metanol (40:60), acetona: cloroformo (1:4), acetona: cloroformo (1:3), dietileter: cloroformo (18:2, Martínez 1972). Las placas se revelaron con los reactivos de Lieberman - Burchard: vainillina en ácido sulfúrico $1 \%$ y cloruro de cobalto en solución de ácido sulfúrico.

Complementariamente se realizaron pruebas de solubilidad de los cristales obtenidos y se determinó el punto de fusión en fusiómetro MEL-TEMP. A la muestra que presentó la mayor pureza en cromatografía de capa fina se le realizaron análisis instru- mentales por resonancia magnética nuclear (RMN de ${ }^{1} \mathrm{H} \mathrm{y}{ }^{13} \mathrm{C}$ e infrarrojo en un espectrómetro ATI Mattson Génesis series FTIR ${ }^{\mathrm{TM}}$. Para el análisis por RMN, la muestra se solubilizó en $10 \mathrm{ml}$ de acetona deuterada y se procesó en un espectrómetro Bruker 400 MHz. El análisis de IR se llevó a cabo en pastilla de $\mathrm{KBr}$.

\section{RESULTADOS}

Los resultados de esta investigación se muestran de acuerdo con el orden del diseño experimental, tanto en laboratorio como en planta piloto. En la Tabla 1 se presentan los promedios en peso de frutos, pericarpio y semillas de jaboncillo, lo mismo que la humedad en \% de tres procedencias: Palmira, Santa María y Viotá. La humedad es una característica importante ya que afecta los costos de producción de cualquiera de los productos.

Tabla 1. Pesos y humedades de frutos, pulpa y semillas de jaboncillo $S$. saponaria de tres procedencias.

\begin{tabular}{cccccccc}
\hline & Fruto & \multicolumn{3}{c}{ Pericarpio-Pulpa } & \multicolumn{3}{c}{ Semilla } \\
\cline { 2 - 8 } Procedencia & $\overline{\mathrm{X}}_{\text {Peso }}$ & $\overline{\mathrm{X}}_{\text {Peso }}$ & \% pulpa & \% humedad & $\overline{\mathrm{X}}_{\text {Peso g }}$ & \% semilla & \% humedad \\
\hline Palmira & 3.36 & 1.85 & 55 & 23.52 & 1.51 & 45 & 10.95 \\
Santa María & 3.016 & 1.596 & 53 & 19.74 & 1.42 & 47 & 8.56 \\
Viotá & 3.048 & 1.83 & 60 & 22.96 & 1.216 & 40 & 8.42 \\
\hline
\end{tabular}

\section{ACEITE}

De las semillas de jaboncillo se extrajo un aceite de buena muy calidad. En la Tabla 2 se presentan los datos correspondientes a los rendimientos de aceite en semillas de tres procedencias. Las propiedades físicas, químicas y organolépticas del aceite refinado de $S$. saponaria se presentan en la Tabla 3. Se pueden observar las principales características que identifican a los aceites comestibles. El color, el sabor y el olor de este aceite cumple con las normas ICONTEC para esta clase de aceite; el índice de yodo indica el grado insaturación de los ácidos grasos contenidos en los esteres de los aceites comestibles. El índice de saponificación indica el grado de disociación de los triglicéridos de los aceites y está correlacionado con el índice de acidez; por último, el índice de refracción identifica el aceite que está directamente relacionado con los porcentajes de ácidos grasos contenidos en los triglicéridos del aceite.

Tabla 2. Rendimiento de aceite en semillas de S. saponaria.

\begin{tabular}{lccc}
\hline \multicolumn{1}{c}{ Procedencia } & Peso semilla $(\mathbf{g})$ & Peso aceite crudo $(\mathbf{g})$ & Rendimiento (\%) \\
\hline Palmira & 54.58 & 2.63 & 4.81 \\
Santa María & 81.76 & 5.78 & 7.06 \\
Viotá & 54.29 & 2.72 & 5.01 \\
\hline
\end{tabular}


Tabla 3. Características físicas, químicas y organolépticas del aceite de $S$. saponaria.

\begin{tabular}{ll}
\hline \multicolumn{1}{c}{ Propiedad } & Resultado \\
\hline Olor & Inoloro \\
Color & Amarillo claro \\
Sabor & Característico \\
Índice de yodo & $118 \mathrm{~g} / 100 \mathrm{~g}$ \\
Acidez & $0.27 \%$ \\
Índice de saponificación & 192 \\
Densidad & $0.95 \mathrm{~g} / \mathrm{ml}$ \\
Índice de refracción & $1.4803 \mathrm{a} 25^{\circ} \mathrm{C}$ \\
\hline
\end{tabular}

En la Tabla 4 se pueden observar los datos correspondientes a la cromatografía de gases (CG), 1 número de señales, tiempos de retención y porcentaje en peso de los correspondientes ácidos grasos presentes en el aceite.

\section{GOMA}

La tabla 5 presenta el contenido de goma en promedio por fruto maduro de $S$. saponaria en Fase 1. En Fase 2, de $6.8 \mathrm{~kg}$ de pulpa madura se obtuvieron dos gomas: una pura (GS), 20.67\%, y una cruda, (EA) 44.98\%. Tanto GS como EA presentaron una viscosidad mayor a 18 centipoises (cps) en una concentración de $2 \%$ en peso a $20^{\circ} \mathrm{C}$, alcanzando un valor de 169 cps y 170 cps en una dilución del $60 \%$, respectivamente. La concentración aproximada de taninos para EA y Gs fue de $1.1 \%$. Las cromatografías de capa fina de EA y GS mostraron que la goma tenía en su estructura glucosa y ramnosa, monosacáridos también presentes en la hederanina extraída de S. saponaria (Abreu 2003). Las características generales de las dos gomas se muestran en la Tabla 6.

Tabla 4. Datos del cromatograma de aceite de jaboncillo $S$. saponaria.

\begin{tabular}{|c|c|c|c|c|}
\hline Señal & Tiempos de retención ( $\mathrm{min}$ ) & $\%$ & & cido graso \\
\hline 1 & 10.583 & 0.13203 & $\mathrm{C} 12: 0$ & Ácido laúrico \\
\hline 2 & 12.176 & 0.06456 & C14: 0 & Ácido mirístico \\
\hline 3 & 14.910 & 5.09022 & C16: 0 & Ácido palmítico \\
\hline 4 & 16.328 & 0.21573 & C16: 1 & Ácido palmitoléico \\
\hline 5 & 18.686 & 4.75617 & C18: 0 & Ácido esteárico \\
\hline 6 & 20.180 & 57.76177 & C18: 1 & Ácido oleico \\
\hline 7 & 20.228 & 3.20235 & C18: 1 & Isómero del ácido oleico \\
\hline 8 & 21.652 & 0.13691 & C18: 2 & Isómero del ácido linoléico \\
\hline 9 & 21.830 & 5.35147 & $\mathrm{C} 20: 0$ & Ácido araquidónico \\
\hline 10 & 22.577 & 9.31966 & C18: 2 & Ácido linoléico \\
\hline 11 & 23.724 & 9.95004 & C18: 3 & Ácido linolénico \\
\hline 12 & 23.887 & 2.06831 & C18: 3 & Isómero del ácido linolénico \\
\hline 13 & 26.055 & 1.14610 & $\mathrm{C} 22: 0$ & Ácido behénico \\
\hline 14 & 27.129 & 0.44227 & \multicolumn{2}{|c|}{ No identificado } \\
\hline 15 & 29.434 & 0.36241 & \multicolumn{2}{|c|}{ No identificado } \\
\hline
\end{tabular}


Tabla 5. Promedio de contenido de goma en fruto maduro de S. saponaria Fase 1.

\begin{tabular}{ccccccc}
\hline \multirow{2}{*}{ Procedencia } & \multicolumn{3}{c}{ Procedimiento A } & \multicolumn{3}{c}{ Procedimiento B } \\
\cline { 2 - 7 } & Pulpa (q) & Goma (g) & $\%$ & Pulpa (g) & Goma (g) & $\%$ \\
\hline Palmira & 5 & 2.35 & 47.0 & 5 & 2.05 & 41.06 \\
Santa María & 5 & 2.53 & 50.7 & 3 & 1.07 & 41.3 \\
Viotá & 5 & 2.353 & 47.06 & 5 & 2.09 & 41.8 \\
\hline
\end{tabular}

Tabla 6. Resultados caracterización de las gomas EA Y GS.

\begin{tabular}{lcc}
\hline \multirow{2}{*}{ Determinación } & \multicolumn{2}{c}{ Goma } \\
\cline { 2 - 3 } & EA & GS \\
\hline Color & Marrón & Marrón \\
Sabor & Amargo & Amargo \\
Olor & Dulce & Dulce \\
\% aproximada taninos & 1.0982 & 1.1972 \\
Alcaloides (Mayer - Dragendorff) & Negativa & Negativa \\
\% humedad & 13.07 & 15.47 \\
\% cenizas & 3.43 & 3.89 \\
\hline
\end{tabular}

\section{SAPOGENINA}

En la fase de laboratorio (Fase 1) se obtuvo el mayor rendimiento del fruto de Viotá aplicando el procedimiento Ps2, 0.11\% (Tabla 7).

Tabla 7. Promedio del contenido de sapogeninas en pulpa verde de S. saponaria fase 1.

\begin{tabular}{cccc}
\hline Procedencia & Procedimiento & No. de muestras & Rendimiento cristales (\%) \\
\hline \multirow{3}{*}{ Palmira } & Ps1 & 3 & - \\
& Ps2 & 3 & 0.08 \\
& Ps3 & 3 & 0.057 \\
\hline \multirow{3}{*}{ Santa María } & Ps1 & 3 & - \\
& Ps2 & 3 & 0.075 \\
& Ps3 & 3 & 0.05 \\
\hline \multirow{3}{*}{ Viotá } & Ps1 & 3 & - \\
& Ps2 & 3 & 0.115 \\
& Ps3 & 3 & 0.07 \\
\hline
\end{tabular}

En el escalado industrial o etapa piloto (Fase 2) el rendimiento de crudo de sapogeninas para PP1 fue de $0.078 \%$ y para PP 2 de $0.047 \%$. De PP 1 se obtuvo la fracción SL1 $1 \mathrm{~g}$ y de PP2 la fracción SL2 $0.58 \mathrm{~g}$. La fracción SL2 no logró purificarse. En la cromatografía de capa fina de sílica gel con mezcla de solventes acetona - cloroformo (1:3) y vainillina en ácido sulfúrico como revelador se pudo observar un Rf y coloración igual para ambas fracciones (Tabla 8). La fracción sL2 no se caracterizó por presentar impurezas. La fracción SL1, cristales blancos amorfos con punto de fusión de $328-330^{\circ} \mathrm{C}$, dio prueba positiva de Lieberman - Bourchard para esteroides y triterpenos. Insoluble en ciclohexano, diclorometano, dietil éter, ácido acético y agua, es medianamente soluble en acetato de etilo, anhídrido acético 
e isopropanol. Soluble en metanol y muy soluble en cloroformo y acetona.

\section{ELUCIDACIÓN ESTRUCTURAL DE LA SUSTANCIA Ss1}

El espectro de IR presentó una banda fuerte y ancha entre 3250 y $3500 \mathrm{~cm}^{-1}$ de un grupo hidroxilo. En $1693 \mathrm{~cm}^{-1}$, banda muy fuerte de estiramiento de $\mathrm{C}=\mathrm{O}$, grupo carboxílico cerca de un doble en- lace. Bandas en 1060 - $800 \mathrm{~cm}^{-1}$ de doble enlace y $643 \mathrm{~cm}^{-1}$, el doblete de la banda en $1642 \mathrm{~cm}^{-1} \mathrm{y}$ un armónico de los cicloalcanos entre 1800 y 2000 $\mathrm{cm}^{-1}$. La banda en $2700 \mathrm{~cm}^{-1}$ con la vibración de la cadena carbonada en $720 \mathrm{~cm}^{-1}$ de anillos alicíclicos juntos, que se confirmó en 1060 y $800 \mathrm{~cm}^{-1}$ y con una banda aguda en $1464 \mathrm{~cm}^{-1}$. Se pudo observar la presencia de $\mathrm{CH}_{3}$ geminales con la banda en 1386 $\mathrm{cm}^{-1}$, confirmándola con el doblete de la banda en $1382-1304 \mathrm{~cm}^{-1}$.

Tabla 8. Datos de los espectros de RMN de ${ }^{1} \mathrm{H}$ y ${ }^{13} \mathrm{C}$ y experimentos HMQC y HMBC.

\begin{tabular}{|c|c|c|c|c|}
\hline Señal & $\delta_{H}(m, J$ en $\mathrm{Hz})$ & $\delta_{c}$ & $\begin{array}{l}\text { HMQC } \\
\text { (C - H) }\end{array}$ & $\begin{array}{l}\text { HMBC } \\
\text { (C- H) }\end{array}$ \\
\hline 1 & $1.053(\mathrm{t}, \mathrm{J}=4.44), 1.622(\mathrm{~s})$ & 38.590 & $1.053,1.622$ & 2 \\
\hline 2 & $1.168(s)$ & 25.167 & 1.168 & \\
\hline 3 & $3.565(d, J=11.6)$ & 75.199 & 3.565 & 1 \\
\hline 4 & - & 43.556 & - & 2,24 \\
\hline 5 & $1.564(\mathrm{t}, \mathrm{J}=4)$ & 49.501 & 1.564 & \\
\hline 6 & $1.416(d, J=4), 1.371(t, J=4)$ & 18.647 & $1.416,1.371$ & \\
\hline 7 & $1.450(d, J=4), 1.371(t, J=4)$ & 32.310 & $1.450,1.371$ & 26 \\
\hline 8 & - & 40.081 & - & \\
\hline 9 & $1.564(\mathrm{t}, \mathrm{J}=4)$ & 47.857 & 1.564 & 26 \\
\hline 10 & - & 38.338 & - & 26 \\
\hline 11 & $1.881(\mathrm{~m})$ & 24.764 & 1.881 & \\
\hline 12 & $5.239(\mathrm{~s})$ & 124.144 & 5.239 & 11 \\
\hline 13 & - & 145.941 & - & 11,16 \\
\hline 14 & - & 43.240 & - & 26 \\
\hline 15 & $2.019(\mathrm{~s}), 1.288(\mathrm{~s})$ & 28.538 & $2.019,1.288$ & 16 \\
\hline 16 & $1.168(s)$ & 25.132 & 1.168 & 27 \\
\hline 17 & - & 48.733 & - & \\
\hline 18 & $3.316(\mathrm{~m})$ & 21.126 & 3.316 & 12,19 \\
\hline 19 & $1.711(\mathrm{~s}), 1.677(\mathrm{~s})$ & 47.792 & $1.711,1.677$ & 29 \\
\hline 20 & - & 29.446 & - & \\
\hline 21 & $1.530(\mathrm{~d}, \mathrm{~J}=4), 1.288(\mathrm{~s})$ & 35.453 & $1.530,1.288$ & 30 \\
\hline 22 & $1.757(\mathrm{t}, \mathrm{J}=4.4), 1.600(\mathrm{~s})$ & 34.265 & $1.757,1.600$ & \\
\hline 23 & $3.793(\mathrm{~m})$ & 69.848 & 3.793 & 25 \\
\hline 24 & $0.939(\mathrm{~d}, \mathrm{~J}=10.4)$ & 17.138 & 0.939 & \\
\hline 25 & $0.745(\mathrm{t})$ & 13.590 & 0.745 & \\
\hline 26 & $0.801(s)$ & 17.334 & 0.801 & \\
\hline 27 & $0.939(d, J=10,4)$ & 27.302 & 0.939 & \\
\hline 28 & - & 179.873 & - & \\
\hline 29 & $0.939(\mathrm{~d}, \mathrm{~J}=10.4)$ & 24.877 & 0.939 & 19 \\
\hline 30 & $0.974(d, J=3.2)$ & 34.359 & 0.974 & \\
\hline
\end{tabular}


En el espectro de RMN ${ }^{1} \mathrm{H}$ (Figura 1) se observan, a campos altos, varias señales de grupos metilo entre 0.5 y $1.0 \mathrm{ppm} \_0.678(\mathrm{~s}, 1 \mathrm{H}), \quad 0.708$ (s, $1 \mathrm{H}), \quad 0.745$ (s, 1H), 0.801 (s, 3H), 0.913 (d, $J$ $=10.4 \mathrm{~Hz}, 9 \mathrm{H}),{ }_{-} 0.974(\mathrm{~d}, J=3.2 \mathrm{~Hz}, 3 \mathrm{H}), 18$ protones de carbonos metílicos. De 1.053 a 1.902 ppm hay señales de protones asociados a carbonos alifáticos secundarios en $\delta 1.053(\mathrm{t}, J=4.4 \mathrm{~Hz} 1 \mathrm{H})$, $\delta 1.168(\mathrm{~s}, 4 \mathrm{H}), \ldots 1.288(\mathrm{~s}, 2 \mathrm{H}),{ }_{1} 1.371(\mathrm{t}, J=4.0$ $H z, 2 \mathrm{H}), \quad 1.416(\mathrm{~d}, J=4.0 \mathrm{~Hz}, 1 \mathrm{H}), \quad 1.450(\mathrm{~d}, J$ $=4.0 \mathrm{~Hz}, 1 \mathrm{H}), \delta 1.530(\mathrm{~d}, J=4.0 \mathrm{~Hz}, \overline{\mathrm{H}}),{ }_{-} 1.600$ $(\mathrm{s}, 1 \mathrm{H}),{ }_{-} 1.622(\mathrm{~s}, 1 \mathrm{H}),{ }_{1} 1.677(\mathrm{~s}, 1 \mathrm{H}),{ }_{-} 1.711(\mathrm{~s}$, $1 \mathrm{H}),{ }_{-} .757$ (t, $\left.J=4.4 \mathrm{~Hz}, 1 \mathrm{H}\right),{ }_{-} 1.881(\mathrm{~m}, 2 \mathrm{H})$, 2.019 (s, 1H), 20 protones. Los protones _1.416 $(\mathrm{d}, J=4.0 \mathrm{~Hz}, 1 \mathrm{H}) \mathrm{y} \_1.450(\mathrm{~d}, J=4.0 \mathrm{~Hz}, 1 \mathrm{H})$ corresponden a enlaces $\mathrm{CH}_{2}-\mathrm{CH}_{2}$. Quintuplete en $2.05 \mathrm{ppm}$ solvente y en $2.875 \mathrm{ppm}$ trazas de agua (Pretsch 1976). En el espectro RMN ${ }^{13} \mathrm{C}$ se observan 30 señales. 5 señales son de CH observadas en DEPT 90,6 son $\mathrm{CH}_{3}$ y $11 \mathrm{CH}_{2}$ determinados por el DEPT 135, dejando 8 señales correspondientes a carbonos cuaternarios. En el espectro de RMN ${ }^{1} \mathrm{H}$ se observa una señal en_5.239 (s, 1H) correspondiente a un protón olefínico que tiene en ${ }^{1} \mathrm{H}-{ }^{1} \mathrm{H}$ cosy una interacción con los protones de señal_1.881 (m, 2H).

El análisis de los espectros permite deducir que la fracción SL1 es un compuesto triterpénico con la presencia de una insaturación en los carbonos 12 y 13, con sustituciones de grupos hidroxilos en los carbonos 3 y 23, así como una sustitución carboxí-

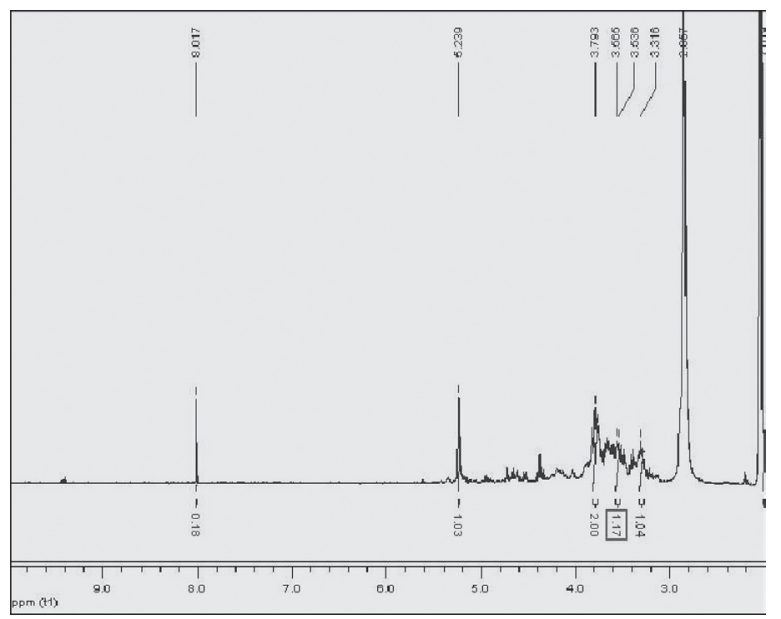

Figura 1. Espectro de RMN ${ }^{1} \mathrm{H}$ de la sustancia Ss1 400 MHZ. lica en el carbono 28, y 6 sustituciones metílicas en los carbonos 10, 8, 14, 20, 20 y 4. La fórmula condensada es $\mathrm{C}_{30} \mathrm{H}_{48} \mathrm{O}_{4}$. En comparación con la simulación realizada para hederagenina con software ACD/CNMR Predictor (v 6.07) y ACD/HNMR Predictor (v 6.08, Escobar \& Medina 2005), se estableció que el compuesto obtenido de la pulpa verde del $S$. saponaria puede corresponder a la Pf. $328-330^{\circ}$ C (Figura 1).

En el espectro RMN ${ }^{13} \mathrm{C}$ (Figura 2) se observa un carbono cuaternario (C28), a 179.873, correspondiente al carbono de un grupo carboxilo ( $\mathrm{COOH})$ que no aparece en los espectros de HMQC, HMBC y COSY debido a que existe una secuencia de pulsos o un retardo que es proporcional al inverso de la constante de acoplamiento ${ }^{13} \mathrm{C}^{1} \mathrm{H}$ y que se ajusta para valores elevados a estos $J_{C H}$ entre 120 y $250 \mathrm{~Hz}$. La señal en _ 69.848 en HMBC (Figura 3) se correlaciona con los protones en $3.793(\mathrm{C} 2)(\mathrm{m}, 2 \mathrm{H})$ indicando tres ligaduras de un metilen a un grupo hidroxilo y se encuentra correlacionado en HMBC con un grupo metilo_ 0.745 (t, 3H) carbono (23). En HMQC el C3 375.199 presenta una correlación a dos ligaduras con grupo metilen_3.565 (d, $J=$ 11.6 $\mathrm{Hz}, 1 \mathrm{H})$, en el espectro HMBC $\overline{\mathrm{C}} 3$ se correlaciona con $\mathrm{C} 1 \mathrm{a}$ tres ligaduras $(\mathrm{C} 1) \_1.053(\mathrm{t}, J=$ $4.441 \mathrm{H})$. En HMQC C1 se encuentra correlacionado a tres ligaduras con protones metílicos en _ 1.053 $(\mathrm{t}, J=4.441 \mathrm{H})$, los protones en $1.622(\mathrm{~s}, 1 \mathrm{H})$ y en HMBC con los protones del C2 y con_1.168 (s 4H) que también es un carbono secundario.

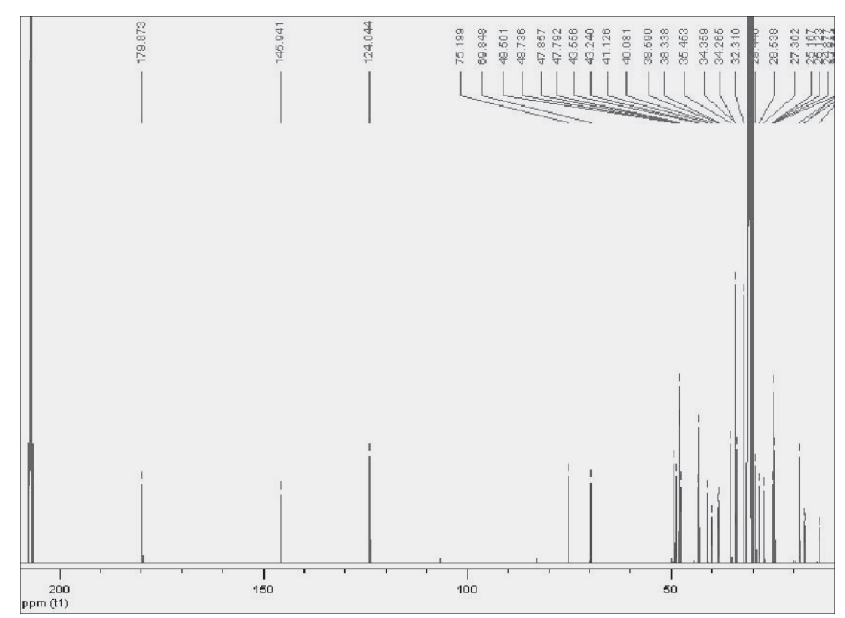

Figura 2. Espectrograma de RMN de $13 \mathrm{C}$ de la sustancia Ss1 a 400 MHZ. 


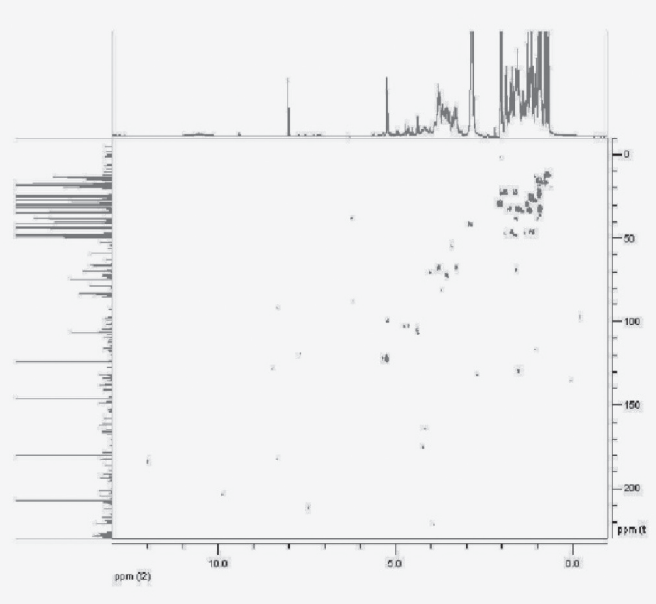

Figura 3. Espectrograma $\mathrm{HMBC}$ de la sustancia Ss1.

De los datos tomados de los espectros de RMN y los experimentos correspondientes se propone la estructura molecular de la Figura 4, correspondiente a la hederagenina, una sustancia que se encontró por primera vez en Hedera hélix, conocida como hiedra, y que se caracteriza por su alta toxicidad en mamíferos. Los extractos de esta planta se utilizan como antitusivos y se comercializan en forma de jarabes.

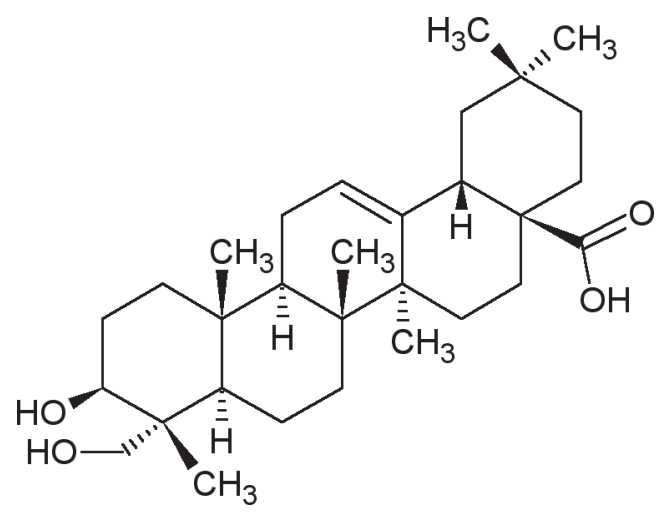

Figura 4. Fórmula estructural de la sustancia Ss1.

\section{DISCUSIÓN}

Los procedimientos seleccionados en Fase 1 fueron replicables y exitosos en la extracción de los tres productos en Fase 2 o etapa piloto, teniendo en cuenta mínimas modificaciones operativas. El aceite obtenido en Fase 2 es semisecante, con un alto contenido de ácidos grasos insaturados $(82.52 \%)$ correspondientes al oleico, linoléico y linolénico (omega 9, 6 y 3 , respectivamente) como en la gran mayoría de aceites saludables. Además de esto, el producto es inodoro, sin turbidez ni sabor característico. Según ICONTEC NTC 218 el valor máximo de acidez permitido para aceite comestible crudo a nivel industrial es de 3\% y para aceites refinados es de $1 \%$. La acidez libre mide el grado de descomposición lipolítica de los glicéridos, este problema se soluciona mediante desodorización del aceite. El aceite de semilla del $S$. saponaria tiene un porcentaje de acidez muy pequeño, lo que significaría una reducción en los costos de refinación. El pequeño porcentaje de isómeros trans (5.3\%) es una factor que hace del aceite una buena opción para la industria alimenticia.

Para la extracción de goma en Fase 1, el procedimiento B no mostró buenos resultados debido a la desnaturalización de la misma en un $\mathrm{pH}$ menor a 2 , valor que afecta la propiedad espesante ya que los enlaces poliméricos se hidrolizan con facilidad. Las propiedades de las gomas EA y GS no presentaron diferencias significativas, indicando con ello que el isopropanol no la purificó, por lo que este paso puede ser eliminado del proceso. Dentro de las sustancias presentes en la goma, y que además se consideran como impurezas difíciles de aislar, están los taninos, que debido a su toxicidad son restringidos en productos alimenticios. La concentración máxima de taninos en gomas para alimentos o medicamentos debe ser de $0.6 \%$ en peso (Anderson 1990). Por tanto, EA podría emplearse en industrias diferentes como espesante de detergentes o pinturas, por sus buenas propiedades viscosantes. A nivel industrial la limpieza de las gomas usadas en productos de consumo humano pasa por procesos de liofilización, diálisis y selección por granulometría, entre otros, dejándolas inodoras y libres de contaminantes (Anderson 1990).

Para sapogeninas en Fase 1, el procedimiento Ps1 dio negativo, probablemente porque la presión en la hidrólisis fue inferior a 1.2 atm, que es la mínima sugerida en el protocolo. Ninguno de los tres procesos sobrepasó el límite de fermentación de 7 días, evitando fermentaciones secundarias que disminuirían el contenido de las sapogeninas (Sharapin 1999). La digestión con sal (Ps3) se aplicó bus- 
cando aumentar la cantidad de material extraíble y controlar la espuma de las saponinas. Sin embargo, la cantidad de sapogeninas obtenidas no aumentó con respecto a Ps2.

En Fase 2, las condiciones de ablandamiento para PP1 fueron favorables, dando mayor rendimiento de precipitado. Se facilitó la purificación de los cristales debido a la interfase de agua, cloroformo y acetona. Del espectro de IR se pudo concluir que la fracción SL1 corresponde a un ácido de naturaleza alicíclica condensado con la presencia de dobles enlaces, grupos metilos geminales y grupos hidroxilos.

\section{CONCLUSIONES}

Los procedimientos aplicados en Fase 1 (laboratorio) fueron replicables a escala piloto (Fase 2), donde se concluyó que la especie $S$. saponaria puede ser aprovechada si este trabajo se complementa con estudios de prefactibilidad industrial.

El diseño y aplicación de procedimientos de extracción a escala piloto para aceite, goma y sapogenina del fruto del $S$. saponaria es viable, teniendo en cuenta los rendimientos.

El aceite extraído de la semilla del fruto del $S$. saponaria podría ser explotado por la industria alimenticia, debido a su alto porcentaje de ácidos grasos insaturados que lo hace saludable y por tener un porcentaje de acidez bajo, lo que implica una reducción en los costos de refinación.

La goma extraída de la pulpa madura del S. saponaria no es apta para la industria alimenticia, farmacéutica o cosmética debido a su contenido de taninos. Sin embargo, sus propiedades viscosantes pueden ser de gran utilidad en otro tipo de industrias, como la de los detergentes, pinturas y/o fertilizantes.

Es posible que la sapogenina obtenida de la pulpa verde del S. saponaria sea la hederagenina, debido a los resultados obtenidos en comparación con las simulaciones de estructura referenciadas para este compuesto.

La extracción de productos a partir de recursos vegetales y su replicación en escala piloto pue- de generar en el país nuevas perspectivas para la investigación en esta área, ampliando la gama de productos derivados de especies promisorias como la estudiada en este proyecto de investigación.

\section{REFERENCIAS BIBLIOGRÁFICAS}

Abed, D. 2002. Caracterización analítica de cinco gomas Mimosaceae Venezolanas y su posible aplicación industrial. Revista facultad de agronomía 19: 230-239.

Abreu, A. 2003. Utilización del fruto del Sapindus saponaria como fuente de saponinas para reducir la metanogénesis y mejorar la utilización del alimento en rumiantes con dietas tropicales. Postgrado - línea de nutrición animal. Facultad de Medicina Veterinaria, Universidad Nacional de Colombia. Pp. 70-75.

Abreu, A., J. E. Carulla, M. Kreuzer, C. E. Lascano, T. E. Díaz, A. Cano \& H. D. Hess. 2003. Efecto del fruto, pericarpio y extracto semipurificado de saponinas de Sapindus saponaria sobre la fermentación ruminal y la metanogénesis in vitro en un sistema RUSITEC. Revista Colombiana de Ciencias Pecuarias 16: 147-154.

Anderson, D., M. Brown, N. Morrison \& W. Weiping. 1990. Specification for gum Arabic (Acacia Senegal), analytical data for samples collected between 1904 and 1989. Food Addit Contamin 7: 303-321.

Baley, A. 1951. Aceites y grasas industriales. Reverté. España. Pp. 72-85.

Escobar, A. \& J. Medina. 2005. Extracción, purificación e identificación de unas sapogeninas presentes en Sapindus saponaria. Escuela de Ingeniería, EAFIT. Medellín. Pp. 6-10.

Hess, H., L. Monsalve, C. Lascano., J. Carulla, T. Díaz \& M. Kreuzer. 2003. Supplementation of a tropical grass diet with forage legumes and Sapindus saponaria fruits: Effects on in vitro ruminal nitrogen turnover and methanogenesis. Australian Journal of Agricultural Research 54: 703-713.

Lemos, T., M. Sousa, L. Mendes \& R. Braz. 1994. Saponin from Sapindus saponaria. Fitoterapia 45: 557-558. 
Marcano, D. \& M. Hesegawa. 1991. Fitoquímica orgánica. Universidad Central de Venezuela. Consejo de desarrollo científico y Humanístico. Pp. 30-40.

Martínez, J. 1972. Análisis orgánico cualitativo. Departamento de Química, Facultad de ciencias, Universidad Nacional de Colombia. Bogotá. Pp. 50-72.

NTC 218. 1999. Método de referencia para la determinación de la acidez. $2^{\mathrm{a}}$ Actualización 1999-06-16.

Pretsch, E., T. Clerc, J. Seibl \& W. Simon. 1976. Tablas para la elucidación estructural de compuestos orgánicos por métodos espectroscópicos. Alhambra. Madrid. Pp. 170-181.

Riveiro, A., C. Zani, T. De Almeida, M. Martineli, M. Hamburger \& K. Hostettmann. 1995. Molluscicidal saponins from the pericarp of
Sapindus saponaria. International Journal of Pharmacognosy 33 (3): 177-180.

Sánchez, J. \& L. Silva. 2008. Estudio silvicultura de la especie Sapindus saponaria L (Jaboncillo o Michú), base de su aprovechamiento Silvoindustrial. Revista Colombia Forestal 11: 79.

Sandoval, J., S. Meza, R. Del Río, G. Hernández, A. Suárez, S. Rincón, N. Farfán \& R. Santillán. 2003. Regioselective cleavage of rings $\mathrm{E}$ and $\mathrm{F}$ in sarsasapogenin. Steroids 68 (2): 199-204.

Sharapin, N. 2000. Fundamentos de tecnología de productos fitoterapeúticos. sapogeninas esteroidales: Materia prima para la fabricación de hormonas esteroidales. Programa Iberoamericanode Ciencia y Tecnología para el Desarrollo (CYTED). Bogotá. Pp. 22-28. 